\title{
Pediatric cerebellar malformations: magnetic resonance diagnostic merits and correlation with neurodevelopmental outcome
}

\author{
Rania S. M. Ibrahim ${ }^{1,2^{*}}$ and Rania H. Hachem ${ }^{1}$
}

\begin{abstract}
Background: In spite of having many classifications for pediatric cerebellar malformations (PCMs), no broadly accepted classification is recommended. Associated neurodevelopmental outcomes in children with PCMs remain poorly defined. Neuroimaging is compulsory for the diagnosis of cerebellar malformation and their associated abnormalities. This article emphasizes on the clinical and radiological traits of PCMs. It proposes a radiological classification and a diagnostic approach and assesses whether specific neuroimaging features in patients with PCM correlate with their neurodevelopmental outcomes.

Results: Fifty-eight pediatric patients were classified as follows: The majority of about 51 cases (88\%) showed cerebellar hypoplasia and the remaining 7 cases (12\%) showed cerebellar dysplasia. Twenty-six patients (45\%) remained undiagnosed, while 32 patients (55\%) were having a final diagnosis (24\% Dandy-Walker malformation (DWM) $(n=14), 7 \%$ isolated vermian hypoplasia $(n=4), 7 \%$ congenital disorder of glycosylation (CDG) $(n=4), 5 \%$ congenital muscular dystrophy $(n=3), 5 \%$ congenital cytomegalovirus (CMV) infection $(n=30), 3 \%$ rhombencephalosynapsis $(n=2)$, 2\% Lhermitte-Duclos syndrome $(n=1)$, and 2\% DWM with Joubert syndrome ( $n$ $=1)$ ). Overall, for the neurodevelopmental outcome, the majority of patients $90 \%(52 / 58)$ had a global developmental delay (GDD) which is a delay in two or more developmental domains. Both motor and language delay represented about 72\% (37/58), intellectual disability was present in 59\% (34/58), epilepsy in 53\% (31/58), ataxic gait in 57\% (33/58), attention deficit hyperactivity disorder (ADHD) in 19\% (11/58), autism spectrum disorder (ASD) in 17\% (10/58), nystagmus and tremors in 15\% (9/58), and behavioral changes in 7\% (6/58). Most of the children with cerebellar hypoplasia, about 93\%, had GDD. Also, patients with PCH associated with a severe GDD, $75 \%$ had a language delay, 50\% had intellectual and motor delay, and about 25\% had epilepsy. However, we observed mild GDD in half of the vermian hypoplasia cases and half of them had a mild fine motor delay.

Conclusions: Magnetic resonance imaging (MRI) of the pediatric brain provides key information to categorize and classify cerebellar malformations. A neurodevelopmental deficit is highly associated with different types of PCMs. Severe GDD was associated with cerebellar and brain stem involvement. However, children with vermis maldevelopment were likely to have mild GDD. Familiarity with their diagnostic criteria is mandatory for correct diagnosis and prognosis for patients.
\end{abstract}

Keywords: Cerebellum, Neuroimaging, Magnetic resonance imaging, Neurodevelopment

\footnotetext{
* Correspondence: raniasaberm@hotmail.com

${ }^{1}$ Radiology Department (Pediatric Unit), Faculty of Medicine, Cairo University,

Kasr Al-aini Street, Al-manial District, Cairo, Egypt

${ }^{2}$ Giza, Egypt
} 


\section{Background}

Radiological classification of pediatric cerebellar malformations (PCMs) is complex with no broadly accepted classification is recommended [1]. They could be classified into inherited (developmental) and acquired (disruptive) abnormalities. A malformation is a congenital morphological aberrance of a single organ or part because of cellular and molecular pathway alteration by gene mutations, teratogens, or combined effects, which is involved in organogenesis. Disruption is an acquired morphological anomaly, which is due to the breakdown of a body structure that had a normal developmental potential that may be caused by, e.g., prenatal infection, hemorrhage, or ischemia. It has a very low recurrence risk [2]. The neurodevelopmental outcomes of PCMs remain poorly defined [3]. Neuroimaging is compulsory for the diagnosis of cerebellar malformation and associated abnormalities. MRI is the neuroimaging tool of choice to assess diagnostic imaging traits in patients with PCMs and correlate with their neurodevelopmental outcomes [4].

\section{Methods}

This is a prospective study that included 58 children patients who were fulfilling the inclusion criteria, attending neurology, neurometabolic outpatient clinics as well as inpatient departments of a pediatric tertiary care hospital (University Children Hospital), during the study period (February 2014 to June 2019). The study protocol was approved by the Research Ethical Committee, University Children Hospital. All patients or patients' guardians were counseled and signed a consent form. Cases were enrolled according to the following inclusion criteria: patients between 1 and 14 years old and suspected to have cerebellar malformation clinically or by cranial ultrasonography. We excluded any cases with other brain abnormalities and otherwise a normal cerebellum. Also, cerebellar atrophy was excluded from the study and any contraindication for MRI examination.

\section{All patients were subjected to the following}

History and clinical examination including general examination and full neurological examination. Laboratory studies are performed. Imaging was done on two devices (Entra and Achieva, Philips MRI system) using a 1.5-T magnet (Table 2); they were imaged in the supine position with the aid of sedation for young patients (chloral hydrate $50-75 \mathrm{mg} / \mathrm{kg}$ orally, 30-60 $\mathrm{min}$ before the procedure). Images included sagittal T1-weighted and sagittal and axial T1- or T2-weighted images. The thickness of the imaging sections was $5 \mathrm{~mm}$. Morphologic features based on MRI findings were recorded for each case. A midline sagittal T1- or T2-weighted sequence is ideal for evaluation of the size of the posterior fossa (which was assessed subjectively), cerebellar hemispheres, and the vermis (folia/fissures pattern) and characteristics (i.e., dysplastic, hypoplastic, or absent) and the size and morphology of the fourth ventricle and brainstem development [4]. Brain MRI was done twice (first for diagnosis and second for follow-up), both were interpreted by two double-blinded experienced pediatric neuroradiologists who were unaware of their clinical features (R.I. and R.H. not less than 10 years of experience). Their neurodevelopmental outcomes and clinical follow-ups were observed by experienced pediatric neurologists along the period from 3 to 5 years. The presence of developmental delay in each domain (gross motor, fine motor, language, and social) and, also, autism spectrum disorder (ASD), intellectual disability, ADHD, seizures, nystagmus, tremors, and ataxic gait were reassessed.

\section{Statistical analysis}

Data management and analysis were performed using the Statistical Package for the Social Sciences, (SPSS 20.0) (Inc., Chicago, Illinois, USA). Quantitative data were summarized using mean and standard deviation and using frequency (count) and relative frequency (percentage).

\section{Results}

Basic demographic data of 58 pediatric patients who included in the present study showed the age of the included patients ranges from 1 to 14 years and the majority, about $48 \%$ of them, was in the first 2 years of life, with male sex predominance $62 \%(n=36)$. The range of follow-up period was 3 to 5 years (mean $=3$ years and 9 months).

Regarding the family history of the included patients, it showed the following: Thirty-six of the studied patients (62\%) were offspring of consanguineous marriages, history of an affected sibling with a neurological disorder which comprised about 35\% of the patients, and history of infant deaths within the family which comprised $60 \%$ of cases.

The main symptoms encountered in the study group were delayed motor development as head support, sitting and walking $(81 \%)$, followed by seizures $(62 \%)$, delayed speech (55\%), and ataxic gait (34\%). Systematic examination of the patients with full neurological examination showed hypotonia and hyporeflexia of about $65 \%$ and $62 \%$, respectively.

Radiological MRI classification of cerebellar malformation revealed the majority $51 / 58$ cases $(88 \%)$ showed cerebellar hypoplasia $(\mathrm{CH})$ which were classified into $50 \%(29 / 58)$ global $\mathrm{CH}, 24 \%(14 / 58) \mathrm{CH}$ with vermian involvement, 7\% (4/58) isolated vermian hypoplasia, and $7 \%(4 / 58)$ pontocerebellar hypoplasia (PCH). The remaining $12 \%$ (7/58) showed cerebellar dysplasia (CD) 
including 5\% (3/58) generalized CD, 5\% (3/58) CD with predominant vermian dysplasia, and $2 \%$ (1/58) predominate hemispheric dysplasia (Table 1).

Regarding their final diagnostic classification, about $45 \%(26 / 58)$ cerebellar malformations remained undiagnosed. However, the majority of cases, $55 \%(32 / 58)$, were having a final diagnosis (24\% Dandy-Walker malformation $(\mathrm{DWM})(n=14)$ represents the most common etiology, $7 \%$ isolated vermian hypoplasia $(n=4), 7 \%$ congenital disorder of glycosylation (CDG) $(n=4), 5 \%$ congenital muscular dystrophy $(n=3), 5 \%$ congenital cytomegalovirus (CMV) infection $(n=3), 3 \%$ rhombencephalosynapsis $(n=2), 2 \%$ Lhermitte-Duclos syndrome $(n=1)$, and $2 \%$ DWM with Joubert syndrome $(n=1))$ (Table 2). A radiological classification of cerebellar malformation by MRI neuroimaging criteria was suggested (Table 3). Overall neurodevelopmental outcome was as follows: the majority of patients, $90 \%$ (52/58), had global developmental delay (GDD) where the majority showing motor and language delay, 72\% (37/58); however, intellectual disability was present in 59\% (34/58), epilepsy was in 53\% (31/58), ataxic gait 57\% (33/58), ADHD in $19 \%(11 / 58)$, ASD in $17 \%(10 / 58)$, nystagmus and tremors in 15\% (9/58), and behavioral changes in 7\% (6/ 58). Most of the children with cerebellar hypoplasia had GDD about 93\%. Also, in patients with $\mathrm{PCH}$ due to CDG associated with a severe GDD, $75 \%$ had a language delay, $50 \%$ had intellectual and motor delay, and about $25 \%$ had epilepsy. However, we observed mild GDD in half of the vermian hypoplasia cases, all patients had language delay, and $75 \%$ had intellectual disabilities and epilepsy. ASD and ADHD were observed in $25 \%$. Half of them had a mild fine motor delay (Table 4).

\section{Discussion}

Cerebellar malformations of the pediatric age group are various groups of cerebellar mal developmental disorders. They have a non-progressive course of clinical, neuroimaging findings and long-term neurodevelopmental outcomes which are still not probably well-defined. A precise analysis of these complex abnormalities is vital for a correct diagnosis and counseling of the family

Table 1 Radiological findings among the studied group

\begin{tabular}{ll}
\hline MRI findings & No. (\%) \\
\hline Global CH & $29(50 \%)$ \\
CH with predominate vermian involvement & $14(24 \%)$ \\
Pontocerebellar hypoplasia & $4(7 \%)$ \\
Isolated vermian hypoplasia & $4(7 \%)$ \\
Generalized cerebellar dysplasia & $3(5 \%)$ \\
Predominantly vermian dysplasia & $3(5 \%)$ \\
Predominantly hemispheric dysplasia & $1(2 \%)$ \\
\hline
\end{tabular}

Table 2 Diagnostic classification of the patients included in the study

\begin{tabular}{ll}
\hline Diagnosis & No. (\%) \\
\hline Cerebellar hypoplasia with unknown diagnosis & $26(45 \%)$ \\
Dandy-Walker malformation (DWM) & $14(24 \%)$ \\
Isolated vermian hypoplasia & $4(7 \%)$ \\
Congenital disorders of glycosylation & $4(7 \%)$ \\
Congenital cytomegalovirus infection & $3(5 \%)$ \\
Congenital muscular dystrophy & $3(5 \%)$ \\
Rhomboencephalosynapsis & $2(3 \%)$ \\
Lhermitte Duclos Cowden Syndrome & $1(2 \%)$ \\
DWM with Jobert syndrome & $1(2 \%)$ \\
TOTAL & $58(100 \%)$ \\
\hline
\end{tabular}

including inheritance pattern, risk of recurrence, and their long term neurodevelopmental outcomes [1].

Cerebellar malformations can be classified into both primary (malformation) and secondary (disruptive) lesions. Primary conditions may be due to chromosomal aberrations (e.g., trisomy 13 and 18), metabolic disorders (e.g., CDG), genetic syndromes (e.g., Joubert syndromes), and, furthermore, congenital brain malformations (primary posterior fossa malformations, e.g., DWM, and rhombencephalosynapsis). Secondary (disruptive) conditions incorporate prenatal infections (e.g., CMV infection), exposure to teratogens, and extreme prematurity. The distinction between malformations and disruptions is important for pathogenesis and genetic counseling [4].

Cerebellar malformations were initially divided into these with hypoplasia and those with dysplasia [5]. $\mathrm{CH}$ refers to cerebellum with a reduced volume, but a normal shape and is stable over time [4]. $\mathrm{CH}$ was classified into (I) focal $\mathrm{CH}$ (unilateral $\mathrm{CH}, \mathrm{CH}$ with mainly vermis involvement, e.g., DWM, and isolated vermian hypoplasia), (II) global $\mathrm{CH}$ with involvement of both vermis and hemispheres (e.g., congenital CMV infection), and (III) $\mathrm{CH}$ with brain stem involvement (e.g., CDG) [6]. Cerebellar atrophy is used if the cerebellum is small with shrunken folia and large cerebellar fissures, or if it has progressive volume loss, patients with cerebellar atrophy were excluded from this study. A CD refers to a disorganized cerebellum with the presence of an abnormal folia pattern or heterotopic nodules of gray matter [5]. CD was classified into (I) focal CD (a. CD with mainly vermis involvement; e.g., Joubert syndrome, and rhombencephalosynapsis; b. predominantly hemispheric dysplasia (e.g., cerebellar cortical dysplasia and Lhermitte-DuclosCowden syndrome) and (II) global CD with the involvement of both vermis and hemispheres (congenital muscular dystrophy) [6] (Table 3).

Fifty-eight pediatric patients were included in this study according to the inclusion criteria with a 
Table 3 Radiological classification of cerebellar malformation by neuroimaging criteria

\begin{tabular}{|c|c|c|c|c|}
\hline \multirow{2}{*}{\multicolumn{3}{|c|}{$\begin{array}{l}\text { Cerebellar hypoplasia } \\
\text { 1. Focal cerebellar hypoplasia }\end{array}$}} & \multicolumn{2}{|l|}{ Cerebellar dysplasia } \\
\hline & & & 1. Focal cerebellar dysplasia & \\
\hline $\begin{array}{l}\text { Isolated vermian } \\
\text { hypoplasia }\end{array}$ & $\begin{array}{l}\text { Predominantly vermian } \\
\text { hypoplasia } \\
\text { DWM }\end{array}$ & $\begin{array}{l}\text { Unilateral hemisphere } \\
\text { hypoplasia }\end{array}$ & $\begin{array}{l}\text { Predominantly vermian } \\
\text { dysplasia } \\
\text { a. Joubert syndrome } \\
\text { b. Rhombencephalosynapsis }\end{array}$ & $\begin{array}{l}\text { Predominantly hemispheric } \\
\text { dysplasia } \\
\text { a. Cerebellar cortical dysplasia } \\
\text { b.(Lhermitte-Duclos-Cowden } \\
\text { syndrome) }\end{array}$ \\
\hline \multicolumn{3}{|c|}{$\begin{array}{l}\text { 2. Global cerebellar hypoplasia } \\
\text { (e.g., congenital CMV infection) }\end{array}$} & \multicolumn{2}{|c|}{$\begin{array}{l}\text { 2. Generalized cerebellar dysplasia } \\
\text { (e.g., congenital muscular dystrophies) }\end{array}$} \\
\hline
\end{tabular}

3. Pontocerebellar hypoplasia (e.g., disorder of glycosylation)

DWM Dandy-Walker malformation, CMV cytomegalovirus

radiological finding of cerebellar malformations. The age of the integrated patients range from 1 to 14 years with the majority, about 28 patients (48\%), of them in the first 2 years of life, with male sex predominance, 36 patients (62\%). Ramaekers et al. [7] reviewed 78 patients (age range, 1 to 16 years) with cerebellar abnormalities, and also D'Arrigo et al. [8] reviewed the clinical and neuroradiological aspects of 51 patients with structural abnormalities of the cerebellum (their age range, 3 months to 14 years and 9 months).

The main presenting symptoms of this study group were GDD $(n=52), 90 \%$ of patients had delayed motor and mental development, seizures was encountered in $(n$ $=36) 62 \%$, delayed speech in $(n=36) 55 \%$, ataxic gait in $(n=20) 34 \%$, an abnormal eye movement in $(n=15)$ $26 \%$, tremors in $(n=9) 15 \%$, and behavioral changes in $(n=6) 10 \%$. Systematic examination of the patients with special attention to the neurology system revealed about $65 \%$ of the patients were with hypotonia and hyporeflexia while about $30 \%$ was normal tone and reflexes, and only $5 \%$ with hypertonia and hyperreflexia. About less than half of the patients $48 \%$ were microcephalic, $25 \%$ were with an ataxic gait, $17 \%$ with nystagmus, and $15 \%$ with abnormal movements.

Regarding MRI findings, the majority 51 cases (88\%) showed $\mathrm{CH}$ and the remaining 7 cases (12\%) showed $\mathrm{CD}$. The group of patients with $\mathrm{CH}$ was classified into the following: 29 patients (50\%) showed global $\mathrm{CH}, 14$ patients $(24 \%)$ with $\mathrm{CH}$ with vermian involvement, 4 patients $(7 \%)$ with isolated vermian hypoplasia, 4 patients (7\%) with $\mathrm{PCH}$. The group having the $\mathrm{CD}$ was classified into the following: generalized $\mathrm{CD}$ in 3 patients (5\%), 3 patients $(5 \%)$ showed predominantly vermian dysplasia, and the last case $(2 \%)$ had predominantly hemispheric

Table 4 Neurodevelopmental outcome in PCMs

\begin{tabular}{|c|c|c|c|c|c|c|c|c|c|c|c|}
\hline \multirow[b]{2}{*}{ PCMs } & \multicolumn{11}{|c|}{ No. cases/\% } \\
\hline & $\overline{\mathrm{GDD}}$ & $\begin{array}{l}\text { Motor } \\
\text { delay }\end{array}$ & $\begin{array}{l}\text { Language } \\
\text { delay }\end{array}$ & $\begin{array}{l}\text { Intellectual } \\
\text { disability }\end{array}$ & ASD & ADHD & Epilepsy & $\begin{array}{l}\text { Ataxic } \\
\text { gait }\end{array}$ & Nystagmus & Tremors & $\begin{array}{l}\text { Behavioral } \\
\text { changes }\end{array}$ \\
\hline $\begin{array}{l}\text { Cerebellar hypoplasia (unknown diagnosis) } \\
(n=29)\end{array}$ & 27 & 21 & 16 & 14 & 0 & 0 & 19 & 6 & 0 & 2 & 3 \\
\hline $\begin{array}{l}\text { Isolated vermian hypoplasia } \\
(n=4)\end{array}$ & 2 & 2 & 4 & 3 & 3 & 2 & 3 & 0 & 0 & 1 & 1 \\
\hline $\begin{array}{l}\text { Dandy-Walker malformation (DWM) } \\
(n=14)\end{array}$ & 12 & 6 & 7 & 8 & 5 & 5 & 3 & 7 & 0 & 3 & 0 \\
\hline $\begin{array}{l}\text { Congenital disorders of glycosylation } \\
(n=4)\end{array}$ & 4 & 2 & 3 & 2 & 1 & 1 & 2 & 4 & 4 & 1 & 1 \\
\hline $\begin{array}{l}\text { Congenital muscular dystrophy } \\
(n=3)\end{array}$ & 3 & 3 & 3 & 3 & 0 & 0 & 2 & 2 & 3 & 1 & 0 \\
\hline $\begin{array}{l}\text { Lhermitte-Duclos Cowden Syndrome } \\
(n=1)\end{array}$ & 1 & 1 & 1 & 1 & 0 & 0 & 1 & 1 & 0 & 0 & 0 \\
\hline $\begin{array}{l}\text { Rhomboencephalosynapsis } \\
(n=2)\end{array}$ & 2 & 1 & 2 & 2 & 1 & 1 & 0 & 2 & 2 & 0 & 1 \\
\hline $\begin{array}{l}\text { DWM with Joubert syndrome } \\
(n=1)\end{array}$ & 1 & 1 & 1 & 1 & 0 & 0 & 1 & 1 & 0 & 1 & 0 \\
\hline Total $(n=58)$ & $\begin{array}{l}52 / \\
90 \%\end{array}$ & $\begin{array}{l}37 / \\
64 \%\end{array}$ & $\begin{array}{l}37 / \\
64 \%\end{array}$ & $\begin{array}{l}34 / \\
59 \%\end{array}$ & $\begin{array}{l}10 / \\
17 \%\end{array}$ & $\begin{array}{l}11 / \\
19 \%\end{array}$ & $\begin{array}{l}31 / \\
53 \%\end{array}$ & $\begin{array}{l}33 / \\
57 \%\end{array}$ & $\begin{array}{l}9 / \\
15 \%\end{array}$ & $\begin{array}{l}9 / \\
15 \%\end{array}$ & $\begin{array}{l}6 / \\
7 \%\end{array}$ \\
\hline
\end{tabular}

$G D D$ global developmental delay, $A S D$ autism spectrum disorder, $A D H D$ attention deficit hyperactivity disorder 
dysplasia. D'Arrigo et al. [8] reviewed the clinical and neuroradiological aspects of 51 patients with structural abnormalities of the cerebellum. Ten had hypoplasia of the vermis, 21 had hypoplasia of the vermis and cerebellar hemispheres, 2 had $\mathrm{PCH}$, and 18 had progressive cerebellar atrophy.

Regarding the etiology and diagnosis of cerebellar malformation, a study done by D'Arrigo et al. [8] stated that 3 patients were diagnosed with CDG type 1a, 2 patients with Cockayne syndrome, and 2 patients with methylmalonic aciduria and homocystinuria. In the current study, $45 \%(n=26)$ had unknown etiology of $\mathrm{CH}$; this was in agreement with Al-Maawali et al. [9] who revealed 158/300 (53\%) of children with cerebellar abnormalities had unknown etiology. For the majority of the patients, about $55 \%(n=32)$, the most common etiology was DWM $24 \%(n=14)$, isolated vermian hypoplasia about 7\% $(n=4)$, CDG about 7\% $(n=4)$, congenital muscular dystrophy $5 \%(n=3)$, congenital CMV infection $5 \%(n=3)$, rhombencephalosynapsis about $3 \%(n=$ $2)$, and DWM with Joubert syndrome $2 \%(n=1)$, and the last case was Lhermitte-Duclos-Cowden syndrome (Table 2).

For the overall neurodevelopmental outcome of the PCMs, we found that $90 \%(52 / 58)$ had GDD; where the majority were showing motor and language delay $72 \%$ (37/58); however, intellectual disability was present in $59 \%(34 / 58)$, ADHD in 19\% (11/58), and ASD in $17 \%$ $(10 / 58)$. However, epilepsy was present in about half of the patients, $53 \%(31 / 58)$. We also observed more outcomes as ataxic gait 57\% (33/58), nystagmus and tremors $15 \%$ (9/58), and behavioral changes 7\% (6/58). This was in concordance with a study done by Pinchefsky et al. [10] found that atypical neurodevelopment was very common, with significant variability in severity between PCM categories. The majority of patients, 82\% (55/67), had GDD where the majority were showing language delay, $88 \%$ (59/67); however, intellectual disability was present in 40\% (27/67), epilepsy in 22\% (15/58), ADHD in $16 \%(11 / 67)$, and ASD in 9\% (6/58). They also found that involvement of the brain stem (such as in $\mathrm{PCH}$ ), and molar tooth malformation (MTM) and the cerebellar hemispheres convey a worse neurodevelopmental prognosis. They suggested that the neurodevelopmental outcome is poorer with $\mathrm{CH}$ than with vermian hypoplasia. In comparison to our study, we agreed with the previous study as radiological evidence of involvement of the brainstem and the cerebellar hemispheres conveys a worse neurodevelopmental prognosis. The presence of $\mathrm{CH}$ and $\mathrm{PCH}$ was significantly associated with severe GDD.

A study done by Pinchefsky et al. [10] found that all children with cerebellar hypoplasia had GDD, being associated with worse motor outcomes, as 74\% (14/19) had a severe motor delay. No patient with cerebellar hypoplasia was diagnosed with ASD, but 71\% (10/14) of children with cerebellar hypoplasia had an intellectual disability. In the current study, most of the children with cerebellar hypoplasia had GDD about 93\%.

The most common etiology of PCMs in current study was DWMs, there were 14 patients (24\%). DWMs occur sporadically and have a low recurrence risk. It may be isolated or part of chromosomal anomalies or Mendelian disorders. Recent genetic studies suggested that DWM may be caused by signaling defects affecting the cerebellum and its overlying mesenchyme [11]. DWM was characterized by neuroimaging by cerebellar and vermian hypoplasia, the elevation of tentorium and torcula, anticlockwise rotation of the vermis, cystic dilatation of the fourth ventricle filling the enlarged posterior fossa, hydrocephalus, brainstem hypoplasia, and associated supratentorial malformations [12]. The neurodevelopmental outcome is variable [13]; it is ranging from typical development to severe cognitive impairment [10]. Although an old study reported that all children with DWM experienced some degree of cognitive impairment $[13,14]$, other studies have reported a more favorable outcome [15]. A study done by Klein et al. [16] found that DWM was associated with ataxia (30-40\%) and cognitive dysfunction. Another study done by Pinchefsky et al. [10] found that DWM had atypical neurodevelopment, with language delay in $100 \%$, GDD in $75 \%$, and intellectual disability in $66 \%$ of those assessed.

In the current study, the majority of patients with DWM had GDD in $86 \%(12 / 14)$, almost near-total to the cases showing motor delay, $43 \%$ (6/14); however, the language delay and intellectual disability represent about $50 \%$ and $64 \%$ respectively, ataxia in $50 \%$ and tremors and epilepsy in $20 \%$.

One case was first diagnosed as DWM showed moderate to severe cerebellar hemispheric hypoplasia and severe vermian hypoplasia and displayed clear evidence of an enlarged posterior fossa with elevation of the torcula associated with mild hydrocephalic changes, but it was associated with elongated, thickened, and horizontally orientated superior cerebellar peduncles (MTM) and a deepened interpeduncular fossa on axial images; the fourth ventricle had "batwing" or triangular configuration and communicated with the cisterna magna, it was diagnosed as DWM with Joubert syndrome (Fig. 1). Joubert syndrome is characterized by the "molar tooth sign" and consists of elongated, thickened, and horizontally oriented superior cerebellar peduncles, a deep interpeduncular fossa, and vermian hypoplasia and dysplasia. Morphologic abnormalities of the brainstem are present in about $30 \%$ of patients and include a dysmorphic tectum and midbrain and thickening and elongation of the midbrain and a small pons. Supratentorial involvement occurs in about $30 \%$ of patients and includes callosal 


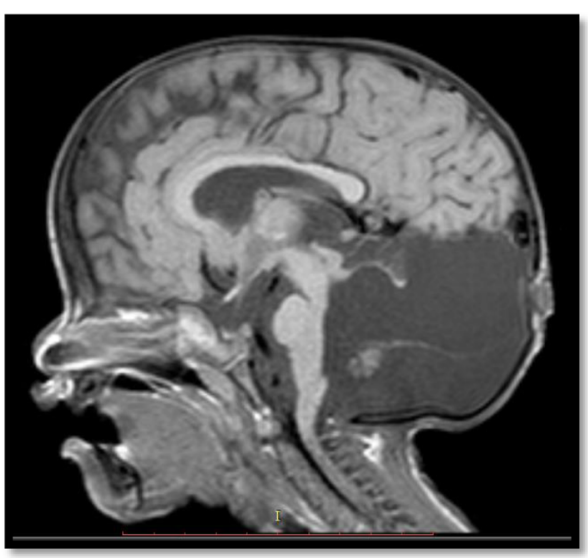

(a)

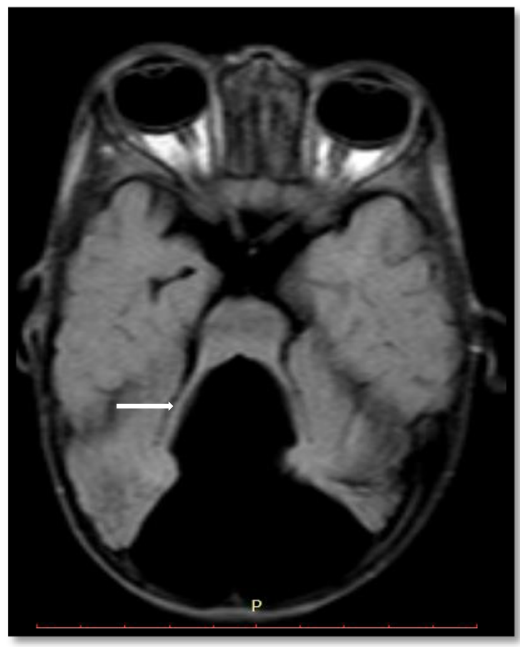

(c)

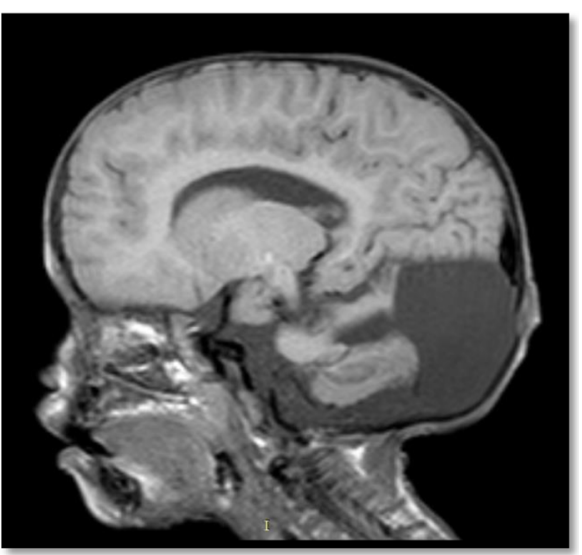

(b)

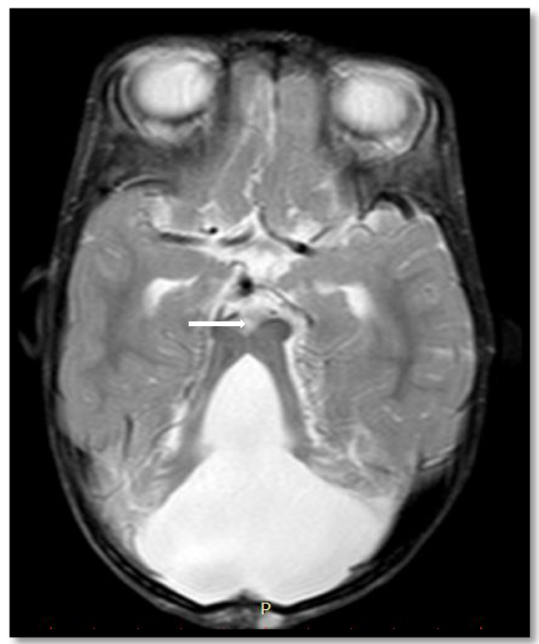

(d)

Fig. $1 \mathrm{MRI}$ of a 3-year-old boy with seizures and delayed motor and mental development. a, b Sagittal and parasagittal T1-weighted images of the brain show cystic dilatation of the fourth ventricle which extends posteriorly filling out nearly the entire posterior fossa, and cerebellar hypoplasia mainly involving the vermis. Elevation of tentorium and torcula with mild hydrocephalic changes. c, d FLAIR and axial T2-weighted image shows the molar tooth sign with elongated, thickened, and horizontally orientated superior cerebellar peduncles (arrows) and a deepened interpeduncular fossa. Final diagnosis: DWM with Joubert syndrome

dysgenesis, cephaloceles, hippocampal mal-rotation, migrational disorders, and ventriculomegaly [17]. Systemic involvement may present and include renal (nephronophthisis), ocular (colobomas, retinal dystrophy), hepatic (congenital hepatic fibrosis), and skeletal (various forms of polydactyly) involvement. Renal and hepatic involvement may cause high morbidity and mortality in patients with Joubert syndrome [18]. It is an autosomal recessive inherited syndrome and with about $25 \%$ recurrence risk in the affected family [18]. A study done by Pinchefsky et al. [10] found that all patients had GDD, motor, and language delay, half of them has an intellectual deficit. In the current study, the only case diagnosed with Joubert syndrome had a severe GDD, with severe motor and language delay, and also he had an intellectual deficit.
In the current study, we diagnosed four patients with isolated vermian hypoplasia who showed partial absence of the inferior vermis. The superior vermis, as well as the cerebellar hemispheres, the fourth ventricle, and the posterior fossa, has a normal size and architecture. Isolated inferior vermian hypoplasia is characterized by a partial absence of the inferior portion of the cerebellar vermis. It has no recurrence risk. More than $75 \%$ of patients with isolated inferior vermian hypoplasia have a favorable outcome. In some patients, mild functional deficits in fine motor activity and receptive language may be present. The prenatal diagnosis of isolated inferior vermian hypoplasia is reliable after 18-20 weeks gestation [19].

Regarding the neurodevelopmental outcome, a study done by Pinchefsky et al. [10] found that 74\% (14/19) of patients with vermis hypoplasia had GDD, most of the 
patients about $89 \%(17 / 19)$ had a language delay. ASD was observed in $31 \%$. ADHD was observed in $47 \%$; they found that patients with vermian hypoplasia had better motor outcomes compared to more severe outcomes observed in the remainder of the PCM studies. Another study by Limperopoulos et al. [18] reported $23 \%$ had delayed development, gross and fine motor disabilities, and social and communication deficits. Furthermore, 15\% of these children were found to have behavioral problems. In the current study, we observed mild GDD in half of the cases, all patients had language delay, and $75 \%$ had intellectual disabilities and epilepsy. Half of them had a mild fine motor delay and ASD and ADHD were observed in $25 \%$.

In this study, we revealed 4 patients with $\mathrm{PCH}$ global cerebellar hypoplasia and pontine hypoplasia (Fig. 2); they were all diagnosed as CDG. PCH is a group of autosomal recessive neurodegenerative disorders with a prenatal onset and is characterized by hypoplasia of the cerebellum and pons [20]. Coronal T2-weighted and sagittal T1- or T2-weighted imaging is fundamental in the evaluation of $\mathrm{PCH}$. On coronal T2-weighted images, it has a "dragonfly" appearance that is created by flattened cerebellar hemispheres (the "wings") and a slightly preserved vermis (the "body") [21]. The morphologic pattern of $\mathrm{PCH}$ is not specific and has also been shown in other selected malformations (e.g., in extreme prematurity) and neurometabolic diseases (e.g., in congenital muscular dystrophies that are caused by defective dystroglycan O-glycosylation) [21]. Patients present with ataxia, nystagmus, postnatal microcephaly, severe cognitive impairment, and sensorineural hearing loss [20]. It includes nearly 50 inborn errors of glycan metabolism. It encompasses defects in $\mathrm{N}$-glycosylation, $\mathrm{O}$-glycosylation, and lipid-linked glycosylation. The most common type is phosphomannomutase II deficiency. Classically, children present with multi-system involvement [22]. In CDG, $\mathrm{PCH}$ may suggest the diagnosis, which must then be confirmed by transferrin electrophoresis and genetic analysis. Treatment for most CDG remains symptomatic and palliative. The prognosis of CDG is variable and depends on the underlying phenotype and genotype [23].

In this study, all patients with PCH due to CDG were significantly associated with a severe GDD, $75 \%$ had a language delay, $50 \%$ had intellectual and motor delay, about $25 \%$ had epilepsy. This was in concordance with a study done by Pinchefsky et al. [10] who found that all children had GDD, which was severe in most of them

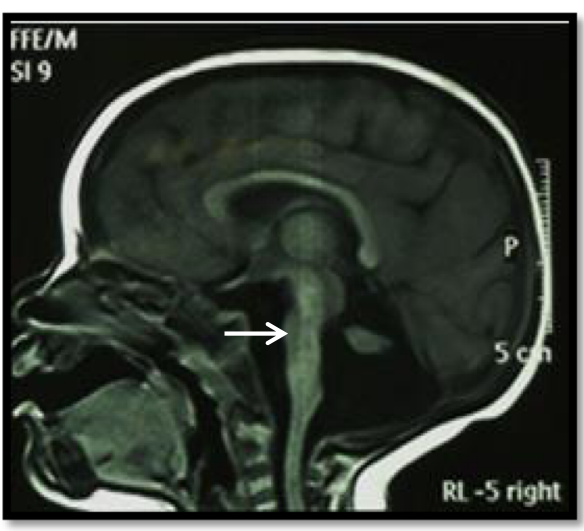

(a)

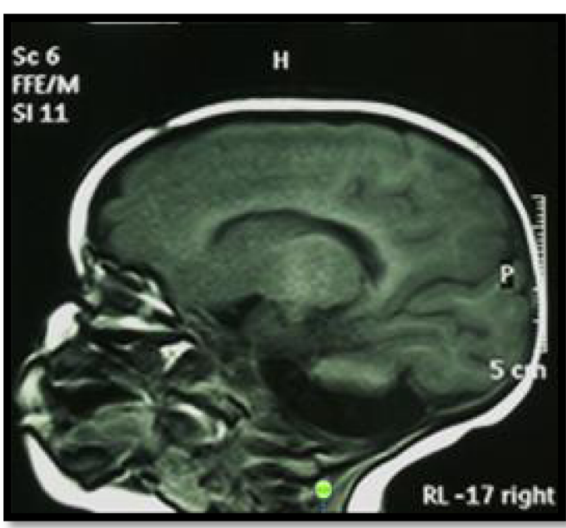

(b)

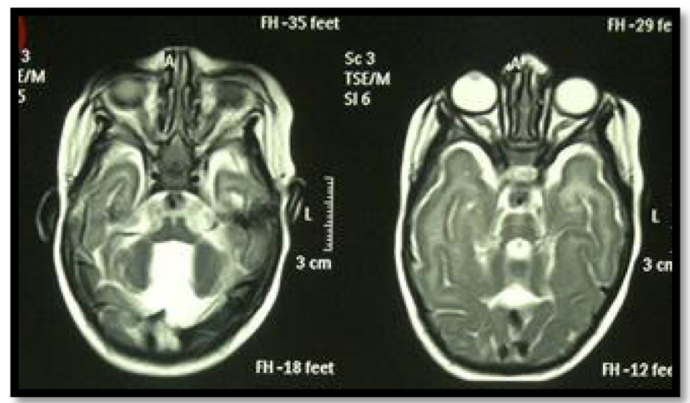

(c)

Fig. 2 A 2.5-year-old female patient with delayed motor and mental development. a, b Mid-sagittal and parasagittal T1-weighted and c axial T2weighted MRI images show global severe cerebellar hypoplasia (marked reduction in size of both cerebellar hemispheres, hypoplasia of inferior cerebellar vermis with relative preservation of the superior midline vermis resulting in a characteristic "dragonfly" appearance) and small pons with flattened anterior surface (arrow). Final diagnosis: pontocerebellar hypoplasia 
$(8 / 9)$ and moderate in one, most of them, $89 \%$, had a language delay, about $33 \%$ had epilepsy.

In the current study, all 3 patients were diagnosed as congenital muscular dystrophy had cortical or subcortical cysts of the cerebellum, multiple small cortical folia, cerebellar polymicrogyria, and cortical dysplasia. Congenital muscular dystrophies (a-dystroglycanopathies) are inherited with an autosomal recessive pattern resulting from mutations in 15 of the genes responsible for the $\mathrm{O}$-glycosylation of a-dystroglycan. The muscles, brain, and eyes are usually affected in a-dystroglycanopathies [24]. Common clinical features are including weakness, hypotonia, contractures, seizures, cognitive impairment, and eye involvement (e.g., retinal dysplasia and micro-ophthalmia) [25]. By MRI, infratentorial involvement; cerebellar hypoplasia or dysplasia, cerebellar cysts, pontine hypoplasia, ventral pontine cleft, and pontomesencephalic kinking are detected. Cerebellar cysts are a specific finding [24]. Supratentorial involvement ranges from mild ventriculomegaly, diffuse periventricular white matter changes and focal areas of polymicrogyria to severe hydrocephalus, generalized white matter signal changes, and diffuse cortical abnormalities, including cobblestone lissencephaly [25]. Regarding neurodevelopmental outcome, all of the four cases showed GDD with severe motor delay, with moderate intellectual and language delay.

We had three cases with global cerebellar hypoplasia with periventricular calcifications; it was diagnosed as congenital CMV infection; all cases showed GDD with language delay, and $67 \%$ had severe motor delay, with

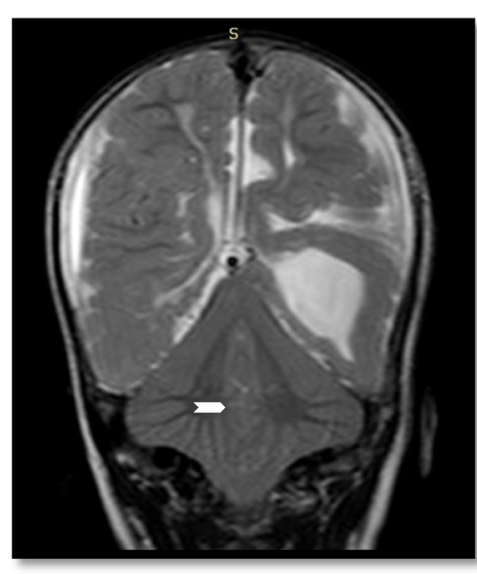

(a)

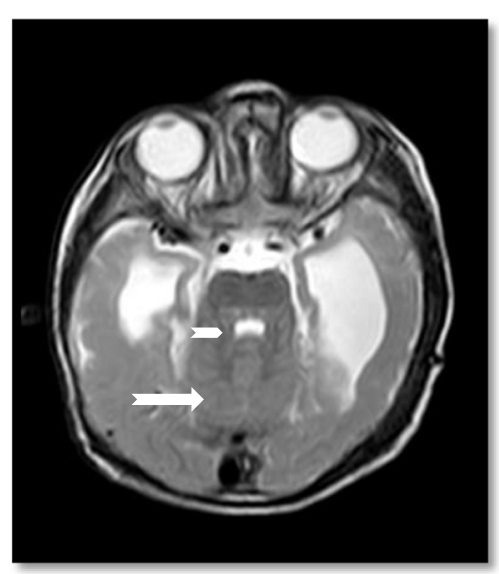

(b)

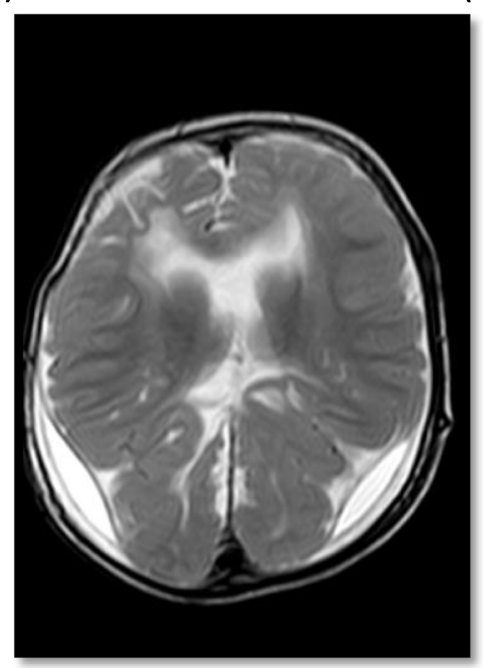

(c)

Fig. 3 A 2.5-year-old boy who presented with ataxia. a Posterior coronal T2-weighted MR image shows continuity of the cerebellar hemispheres with an abnormal transverse orientation of the cerebellar foliae (arrowheads). b, c Axial T2-weighted MR image shows continuity of the cerebellar hemispheres, dentate nuclei (arrows), and superior cerebellar peduncles without an intervening vermis, key hole appearance of the fourth ventricle (arrowheads), associated with aqueductal stenosis and supratentorial abnormalities (supratentorial hydrocephalus, absent septum pellucidum, callosal dysgenesis, abnormal gyration, and also bilateral subdural hematoma). Final diagnosis: rhomboencephalosynapsis 
moderate intellectual disability. CMV infection results in periventricular calcifications and cerebellar hypoplasia with the thin cortex and shallow sulci [26].

Two patients with rhombencephalosynapsis had been diagnosed in this study, characterized by the absence of the cerebellar vermis with midline fusion of the two cerebellar hemispheres. The dentate nuclei and superior cerebellar peduncles were small and appeared fused. The cerebral hemispheres showed subjectively reduced white matter volume, colpocephaly, and hypogenesis of corpus callosum and absence of the septum pellucidum, a dysplastic cerebral cortex, most likely polymicrogyria, and bilateral subdural hematoma. The volume of the brain stem was also subjectively reduced (Fig. 3). A study done by Pinchefsky et al. [12] found that the outcome included mild GDD, borderline intellectual disability, and ADHD in one individual and moderate gross motor and fine motor delays in the other. In the current study, both patients diagnosed as rhombencephalosynapsis had a mild GDD, ataxic gait, mild language and intellectual disability, and nystagmus. However, only one of them had ADS, ADHD, and motor delay. The majority of patients diagnosed as rhombencephalosynapsis are non-syndromic. The most common clinical manifestations include truncal and/or limb ataxia, abnormal eye movements, head stereotypies, and delayed motor development. The key neuroimaging findings are agenesis of the vermis and continuity of the cerebellar hemispheres, superior cerebellar peduncles, and dentate nuclei, which creates a horseshoe-shaped arch across the midline (a keyholeshaped fourth ventricle)). Posterior coronal T2-weighted images are crucial in showing the horizontal folia pattern, whereas the mid-sagittal T1-weighted image depicts the dentate nuclei. Rhombencephalosynapsis may be associated with other central nervous system anomalies such as hydrocephalus, mostly due to aqueduct stenosis and forebrain abnormalities including absent olfactory bulbs, dysgenesis of the corpus callosum, and absent septum pellucidum. The long-term cognitive outcome varies from severe impairment to normalcy [27].

The last patient showed a sharply defined, nonenhancing focal cerebellar mass in the left cerebellar hemisphere being hypointense in $\mathrm{T} 1$ and hyperintense in $\mathrm{T} 2$ relaxation times, with isointense curvilinear stripes. The mass resulted in a moderate mass effect on the brain stem. Considering that the patient had hamartomata of the face and multiple other organs and, thus, had the diagnosis of Cowden syndrome, a confident diagnosis of dysplastic gangliocytoma of the cerebellum was made. The cerebral hemispheres were otherwise normal. The follow-up showed a mild GDD. Lhermitte-Duclos disease (dysplastic cerebellar gangliocytoma) is probably hamartomatous. It is typically presented in young adults. It is associated with other conditions including disorders of cortical formation, megalencephaly, grey matter heterotopia, polymicrogyria, polydactyly, hydromyelia, macroglossia, and localized gigantism. Small lesions may be asymptomatic. However, large lesions may manifest as raised intracranial pressure, obstructive hydrocephalus, and to a lesser degree, cerebellar dysfunction. By MRI, it typically shows non-enhancing focal enlargement of cerebellum with prolonged $\mathrm{T} 1$ and $\mathrm{T} 2$ relaxation times with widened cerebellar folia with a striated/ tigroid appearance in T2. Enhancement is rare. It shows elevated lactate, slightly reduced $\mathrm{N}$-acetyl aspartate, reduced myoinositol, reduced choline, and reduced choline/creatine ratio. It has a strong association with visceral hamartomas. The dysplastic mass grows very slowly. Surgical resection is often curative, with only a few case reports of recurrence [28].

The reason for the variability of the neurodevelopmental outcome in between studies may reflect the small sample size of the included studies and referral bias, and a high degree of variability in the definition of the different PCMs leads to misclassification of the patients; at last, the differences in age at follow-up represent the reasons. Also, a basic neurological examination may not be sufficient to determine the neurodevelopmental status of these children and more accurate tests investigating cognitive, affective and behavioral functions are needed to ascertain the actual rate of abnormal development. Also, a future study for PCMs is needed to highlight the additional information that new MRI techniques as diffusion tensor or volumetric study may provide compared with conventional MR imaging sequences.

\section{Conclusions}

Magnetic resonance imaging of the pediatric brain provides key information to categorize and classify cerebellar malformations. Long-term neurodevelopmental deficits are highly associated with different types of PCMs. Severe GDD was significantly associated with cerebellar and brain stem involvement. However, children with vermis mal-development were likely to have mild GDD. Familiarity with their diagnostic criteria is mandatory for a correct diagnosis, recurrence risk counseling, and prognosis for patients.

\section{Abbreviations \\ ADHD: Attention deficit hyperactivity disorder; ASD: Autism spectrum disorder; CD: Cerebellar dysplasia; CDG: Congenital disorder of glycosylation; $\mathrm{CH}$ : Cerebellar hypoplasia; CMV: Cytomegalovirus; DWM: Dandy-Walker malformation; GDD: Global developmental delay; MRI: Magnetic resonance imaging; MTM: Molar tooth malformation; PCMs: Pediatric cerebellar malformations}

\section{Acknowledgements}

The authors wish to acknowledge the assistance of pediatric neurology department staff, for their contribution to patient data collection.

\section{Authors' contributions}

Rl contributed to the study concept, study design, data acquisition, manuscript preparation, and manuscript editing. $\mathrm{RH}$ and $\mathrm{RI}$ contributed to 
the data analysis and interpretation, statistical analysis, and manuscript reviewing. All authors have read and approved the manuscript.

\section{Funding}

Self-funding.

\section{Availability of data and materials}

The datasets used and/or analyzed during the current study are available from the corresponding author on reasonable request.

\section{Ethics approval and consent to participate}

This study was approved by Radiology Department Kasr Alaini Cairo University hospital research ethics committee. Number: not available. Written informed consent was signed by the patients' guardians.

\section{Consent for publication}

All patients' guardians included in this research gave written informed consent to publish the data contained within this study. If the patient was less than 16 years old, deceased, or unconscious when consent for publication was requested, written informed consent for the publication of this data was given by their parent or legal guardian.

\section{Competing interests}

The authors declare that they have no competing interests.

Received: 22 October 2019 Accepted: 17 February 2020

Published online: 04 March 2020

\section{References}

1. Patel S, Barkovich AJ (2002) Analysis and Classification of Cerebellar Malformations. AJNR Am J Neuroradiol 23:1074-1087

2. Hennekam RC, Biesecker LG, Allanson JE, Hall JG, Opitz JM, Temple IK et al (2013) Elements of morphology: general terms for congenital anomalies. Am J Med Genet A. 161A(11):2726-2733

3. D'Antonio F, Khalil A, Garel C, Pilu G, Rizzo G, Lerman-Sagie T et al (2016) Systematic review and meta-analysis of isolated posterior fossa malformations on prenatal imaging (part 2): neurodevelopmental outcome. Ultrasound Obstet Gynecol 48:28-37

4. Poretti A, Boltshauser E, Doherty D (2014) Cerebellar hypoplasia: differential diagnosis and diagnostic approach. Am J Med Genet Part C 166C:211-226

5. Barkovich AJ (2000) Congenital malformations of the brain and skull. In: Barkovich AJ (ed) Pediatric Neuroimaging, 3rd edn. Lippincott Williams \& Wilkins, Philadelphia, pp 251-381

6. Arora R (2015) Imaging Spectrum of Cerebellar Pathologies: A Pictorial Essay. Pol J Radiol 80:142-150

7. Ramaekers VT, Heimann G, Reul J, Thron A, Jaeken J (1997) Genetic disorders and cerebellar structural abnormalities in childhood. Brain 120(10): 1739-1751

8. D'Arrigo S, Viganò L, Grazia Bruzzone M, Marzaroli M, Nikas I, Riva D et al (2005) Diagnostic approach to cerebellar disease in children. J Child Neurol. 20(11):859-866

9. Al A-M, Blaser S, Yoon G (2012) Diagnostic approach to childhood-onset cerebellar atrophy: a 10-year retrospective study of 300 patients. J Child Neurol 27(9):1121-1132

10. Pinchefsky EF, Accogli A, Shevell MI, Martin CHS, Srour M (2019) Developmental outcomes in children with congenital cerebellar malformations. Dev Med Child Neurol 61:350-385

11. Alexiou GA, Sfakianos G, Prodromou N (2010) Dandy-Walker malformation: analysis of 19 cases. J Child Neurol. 25(2):188-191

12. Bosemani T, Orman G, Boltshauser E, Tekes A, Huisman TA, Poretti A (2015) Congenital abnormalities of the posterior fossa. Radiographics 35(1):200-220

13. Boddaert $N$, Klein $O$, Ferguson $N$, Sonigo P, Parisot D, Hertz-Pannier L et al (2003) Intellectual prognosis of the Dandy-Walker malformation in children: the importance of vermian lobulation. Neuroradiology 45(5):320-324

14. Aletebi F, Fung K (1999) Neurodevelopmental outcome after antenatal diagnosis of posterior fossa abnormalities. J Ultrasound Med 18:683-689 20. Klein O, Pierre-Kahn A, Boddaert N

15. Parisot D, Brunelle F (2003) Dandy Walker malformation: prenatal diagnosis and prognosis. Childs Nerv Syst 19:484-489

16. Brancati F, Dallapiccola B, Valente EM (2010) Joubert syndrome and related disorders. Orphanet J Rare Dis 5:20
17. Poretti A, Huisman TA, Scheer I, Boltshauser E (2011) Joubert syndrome and related disorders: spectrum of neuroimaging findings in 75 patients. AJNR 32(8):1459-1463

18. Romani M, Micalizzi A, Valente EM (2013) Joubert syndrome: congenital cerebellar ataxia with the molar tooth. Lancet Neurol 12(9):894-905

19. Limperopoulos C, Robertson RL, Estroff JA, Barnewolt C, Levine D, Bassan H et al (2006) Diagnosis of inferior vermian hypoplasia by fetal magnetic resonance imaging: potential pitfalls and neurodevelopmental outcome. Am J Obstet Gynecol 194(4):1070-1076

20. Namavar $Y$, Barth PG, Kasher PR, van Ruissen F, Brockmann K, Bernert $G$ et al (2011) Clinical, neuroradiological and genetic findings in pontocerebellar hypoplasia. Brain 134(1):143-156

21. Najm J, Horn D, Wimplinger I, Golden JA, Chizhikov W, Sudi J et al (2008) Mutations of CASK cause an X-linked brain malformation phenotype with microcephaly and hypoplasia of the brainstem and cerebellum. Nat Genet 40(9):1065-1067

22. Freeze HH, Chong JX, Bamshad MJ, Ng BG (2014) Solving glycosylation disorders: fundamental approaches reveal complicated pathways. Am J Hum Genet. 94(2):161-175

23. Klein JL, Lemmon ME, Northington FJ, Boltshauser E, HuismanTH AGM, Poretti A (2016) Clinical and neuroimaging features as diagnostic guides in neonatal neurology diseases with cerebellar involvement. Cerebellum Ataxias. 3:1

24. Clement E, Mercuri E, Godfrey C, Smith J, Robb S, Kinali M et al (2008) Brain involvement in muscular dystrophies with defective dystroglycan glycosylation. Ann Neurol 64(5):573-582

25. Godfrey C, Clement E, Mein R, Brockington M, Smith J, Talim B et al (2007) Refining genotype phenotype correlations in muscular dystrophies with defective glycosylation of dystroglycan. Brain 130(10):2725-2735

26. De Vries LS, Gunardi H, Barth PG, Bok LA, Verboon-Maciolek MA, Groenendaal F (2004) The spectrum of cranial ultrasound and magnetic resonance imaging abnormalities in congenital cytomegalovirus infection. Neuropediatrics 35(2):113-119

27. Ishak GE, Dempsey JC, Shaw DW, Tully H, Adam MP, Sanchez-Lara PA et al (2012) Rhombencephalosynapsis: a hindbrain malformation associated with incomplete separation of midbrain and forebrain, hydrocephalus and a broad spectrum of severity. Brain 135(5):1370-1386

28. Klisch J, Juengling F, Spreer J, Koch D, Thiel T, Büchert M et al (2001) Lhermitte-Duclos disease: assessment with MR imaging, positron emission tomography, single-photon emission CT, and MR spectroscopy. AJNR Am J Neuroradiol. 22(5):824-830

\section{Publisher's Note}

Springer Nature remains neutral with regard to jurisdictional claims in published maps and institutional affiliations.

\section{Submit your manuscript to a SpringerOpen ${ }^{\circ}$ journal and benefit from:}

- Convenient online submission

- Rigorous peer review

- Open access: articles freely available online

- High visibility within the field

- Retaining the copyright to your article

Submit your next manuscript at $>$ springeropen.com 